\title{
Novos insights sobre a associação entre índice de massa corporal e COVID-19 grave.
}

\author{
Jamille dos Passos Lacerda (1) e Éber Coelho Paraguassu (1).
}

\section{EDITORIAL}

Resumo

A emergente pandemia SARS-CoV-2 chamou a atenção mundial nos primeiros meses de 2020 . A maioria das pessoas que são infectadas tem doença leve ou são assintomáticas, mas muitas têm doenças graves e frequentemente fatais. A idade avançada rapidamente emergiu como o fator de risco mais importante para desfechos desfavoráveis, mas a obesidade foi um de vários outros fatores rapidamente identificados como associados à mortalidade associada ao COVID-19.

Palavras Chave: Covid-19, IMC, Obesidade. 


\title{
New insights into the association between body mass index and severe COVID-19.
}

\begin{abstract}
The emerging pandemic SARS-CoV-2 drew worldwide attention in the first months of 2020. Most people who are infected have mild illness or are asymptomatic, but many have serious and often fatal illnesses. Advanced age quickly emerged as the most important risk factor for unfavorable outcomes, but obesity was one of several other factors quickly identified as associated with COVID-19-associated mortality.
\end{abstract}

Keywords: Covid-19, BMI, Obesity.

Instituição afiliada: 1- GOE/Uniavan.

Dados da publicação: Artigo recebido em 10 de Abril, revisado em 15 de Abril, aceito para

publicação em 20 de Abril e publicado em 28 de Março.

DOI: https://doi.org/10.36557/2674-8169.2021v3n2p01-06

Éber Coelho Paraguassu paraguassutans@gmail.com

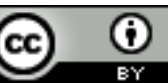

This work is licensed under a Creative Commons Attribution 4.0 International

License. 


\section{COVID $19 \times$ IMC ALTO}

A emergente pandemia SARS-CoV-2 chamou a atenção mundial nos primeiros meses de 2020. $\stackrel{1}{\text { A }}$ maioria das pessoas que são infectadas tem doença leve ou são assintomáticas, mas muitas têm doenças graves e frequentemente fatais. $A$ idade avançada rapidamente emergiu como o fator de risco mais importante para desfechos desfavoráveis, mas a obesidade foi um de vários outros fatores rapidamente identificados como associados à mortalidade associada ao COVID-19. ${ }^{2}$

No The Lancet Diabetes \& Endocrinology, Min Gao, Carmen Piernas e colegas ${ }^{3}$ apresentam pesquisas detalhadas sobre o risco de desfechos COVID-19 graves em pessoas com IMC alto. Este estudo abrangente baseia-se em evidências anteriores, examinando uma gama de resultados em todo o espectro do IMC. Usando o banco de dados QResearch de registros eletrônicos de atenção primária na Inglaterra, Reino Unido, vinculados aos resultados do teste SARS-CoV-2, admissões hospitalares e dados de registro de óbitos, Gao e colegas identificaram mais de 6,9 milhões de indivíduos com pelo menos um registro de IMC com 20 anos anos e mais velhos que tinham dados disponíveis no sistema QResearch entre 24 de janeiro e 30 de abril de 2020, entre os quais 13.503 internações hospitalares, 1.601 internações em unidade de terapia intensiva (UTI) e 5.479 mortes devido a COVID-19 ocorreram no período do estudo ( corte de dados em 30 de abril de 2020). Associações em forma de J foram observadas entre o IMC e as admissões hospitalares e óbitos do COVID-19; ${ }^{2}$, enquanto o aumento no risco de morte começou a aumentar linearmente com um IMC ligeiramente superior de $28 \mathrm{~kg} / \mathrm{m}^{2}$, em conformidade com a associação entre o IMC e uma ampla gama de desfechos de mortalidade por causa específica. ${ }^{4}$

Foi observada uma forte interação com a idade, com o aumento da unidade no IMC sendo associado a grandes aumentos no risco para o grupo de idade mais jovem (20-39 anos), e nenhuma associação no grupo de idade mais velha ( $\geq 80$ anos).

Os autores também identificaram um risco aumentado de internação hospitalar e morte devido ao COVID-19 nos níveis de IMC mais baixos, o que é um novo insight não abordado pela pesquisa inicial do COVID-19 que focava na obesidade. Uma limitação da análise apresentada por Gao e colegas é que as interações foram avaliadas apenas naqueles com IMC de $23 \mathrm{~kg} / \mathrm{m}^{2}$ ou superior, de forma que a associação entre baixo peso e risco em grupos de idade mais avançada (nos quais o baixo IMC é comum) não pôde ser discernida. Curiosamente, em contraste com outros resultados, o IMC foi aproximadamente linearmente associado à admissão na UTI em toda a faixa de IMC, sem aumento no risco de IMC baixo. Esta observação não é fácil de interpretar. A admissão na UTI reflete não apenas a doença grave, mas também o complexo processo 
de tomada de decisão clínica sobre a probabilidade de um paciente tolerar e se beneficiar de um tratamento intensivo. ${ }^{5}$

O risco reduzido de admissão à UTI em indivíduos com baixo peso provavelmente reflete a condição de pacientes com baixo peso de maior risco considerados inadequados para tratamento intensivo.

Outra limitação importante do estudo é que mais de um milhão de indivíduos não tinham registro de IMC disponível e foram excluídos das análises principais. A disponibilidade de muitos milhões de registros de IMC coletados rotineiramente é um ponto forte das fontes de dados de cuidados primários do Reino Unido, mas a falta de dados é um problema comum, especialmente porque o IMC pode ter maior probabilidade de ser registrado se estiver fora da faixa de peso saudável, violando o chamado faltando ao acaso a suposição necessária para as abordagens populares de imputação múltipla. ${ }^{6}$

Gao e colegas devem ser elogiados por fornecer dados descritivos sobre esses indivíduos, entre os quais uma proporção maior era jovem (com idade entre 20 e 39 anos) e do sexo masculino do que na população com medidas de IMC. A restrição das análises àqueles com dados completos pode ser uma abordagem razoável, mesmo se eles não forem totalmente representativos da população mais ampla de pacientes, desde que a associação entre o IMC e os resultados sejam corretamente representada pelos incluídos. ${ }^{7}$

Outra limitação importante é que a investigação de desfechos graves de COVID19 em uma coorte populacional geral não permite distinguir se o IMC está associado ao risco de infecção, progressão para doença grave após a infecção ou ambos. As hipóteses mecanísticas até o momento sugeriram que um desequilíbrio nas citocinas próinflamatórias e anti-inflamatórias com IMC mais alto pode promover doença COVID-19 mais grave do que naqueles com IMC mais baixo. ${ }^{8}$

No entanto, é improvável que qualquer estudo epidemiológico usando dados de grande escala disponíveis seja capaz de isolar de forma confiável a associação entre o IMC e os resultados em indivíduos infectados, porque isso exigiria dados de teste representativos em grande escala para identificar infecções assintomáticas e sintomáticas. Na realidade, o teste COVID-19 no Reino Unido e na maioria dos outros ambientes tem sido sistematicamente direcionado para aqueles com sintomas, tornando um teste positivo um marcador insensível e seletivo de infecção, e aqueles com teste positivo uma amostra tendenciosa para examinar os resultados da doença grave. Por razões semelhantes, Gao e seus colegas evitaram, com razão, um enfoque nos resultados entre os pacientes que já estavam no hospital. ${ }^{9}$ 
Pessoas com um IMC de mais de $40 \mathrm{~kg} / \mathrm{m}^{2}$ foram reconhecidas no início das orientações de saúde pública como estando em risco aumentado de desfechos COVID19 graves, e o IMC agora está sendo usado em ferramentas de previsão de risco que informam a priorização de vacinas, ${ }^{10}$ destacando a importância de obter uma compreensão detalhada da associação entre o IMC e os resultados do COVID-19 para informar a política. As principais prioridades de pesquisas futuras serão estabelecer se - IMC afeta a eficácia da vacina e compreender se as pessoas fora da faixa de IMC consideradas saudáveis $\left(18,5-24,9 \mathrm{~kg} / \mathrm{m}^{2}\right)$ apresentam risco aumentado de pósCOVID-19 sequelas. Um estudo epidemiológico cuidadoso dessas e de outras questões emergentes informará a resposta contínua da saúde pública a esta nova doença que provavelmente veio para ficar. ${ }^{11}{ }^{12},{ }^{13},{ }^{15}, 16$.

\section{THE AUTHORS DECLARE NO CONFLICTS OF INTEREST.}

\section{REFERÊNCIAS}

1. WHO. WHO Director-General's opening remarks at the media briefing on COVID-19 11 March 2020. World Health Organization, March 11, 2020. https://www.who.int/dg/speeches/detail/who-director-general-s-opening-remarks-atthe-media-briefing-on-covid-19-11-march-2020

2. Williamson EJ, Walker AJ, Bhaskaran K, et al. Factors associated with COVID-19-related death using OpenSAFELY. Nature. 2020; 584: 430-436

3. Gao M, Piernas C, Astbury N, et al. Associations between body-mass index and COVID19 severity in 6.9 million people in England: a prospective, community-based cohort study. Lancet Diabetes Endocrinol. 2021; (published online April 28.) https://doi.org/10.1016/S2213-8587(21)00089-9

4. Bhaskaran K, Dos-Santos-Silva I, Leon DA, Douglas IJ, Smeeth L. Association of BMI with overall and cause-specific mortality: a population-based cohort study of 3.6 million adults in the UK. Lancet Diabetes Endocrinol. 2018; 6: 944-953

5. Griffiths $F$, Svantesson M, Bassford $C$, et al. Decision-making around admission to intensive care in the UK pre-COVID-19: a multicentre ethnographic study. Anaesthesia. 2021; 76: 489-499.

6. Bhaskaran $\mathrm{K}$, Smeeth $\mathrm{L}$. What is the difference between missing completely at random 
and missing at random?. Int J Epidemiol. 2014; 43: 1336-1339

7. White IR, Carlin JB. Bias and efficiency of multiple imputation compared with completecase analysis for missing covariate values. Stat Med. 2010; 29: 2920-2931.

8. Post A, Bakker SJL, Dullaart RPF. Obesity, adipokines and COVID-19. Eur J Clin Invest. 2020; 50e13313

9. Griffith GJ, Morris TT, Tudball MJ et al. Collider bias undermines our understanding of COVID-19 disease risk and severity. Nat Commun. 2020; 115749.

10. Clift AK, Coupland CAC, Keogh RH, et al. Living risk prediction algorithm (QCOVID) for risk of hospital admission and mortality from coronavirus 19 in adults: national derivation and validation cohort study. BMJ. 2020; 371m3731

11. Coelho Paraguassu, E. ., Chen, H. ., Zhou, F. ., Xu , Z. ., \& Wang, M. . (2020). Coronavirus and COVID-19: The latest news and views from the scientific community about the new coronavirus and COVID-19. Brazilian Journal of Implantology and Health Sciences , 2(3), 96-109. https://doi.org/10.36557/2674-8169.2020v2n3p96-109.

12. Athayde, A., \& Silva, M. F. (2021). COVID-19: Tendências em mudança e seu impacto no futuro da odontologia. Brazilian Journal of Implantology and Health Sciences , 3(2), 11 23. https://doi.org/10.36557/2674-8169.2021v3n2p11-23.

13. Lemos, P. ., de Almeida Filho, N. ., \& Firmo, J. (2020). COVID-19, desastre do sistema de saúde no presente e tragédia da economia em um futuro bem próximo. Brazilian Journal of Implantology and Health Sciences, 2(4), 39-50. https://doi.org/10.36557/26748169.2020v2n4p39-50.

14. Coelho Paraguassu, E. ., \& Celis de Cárdenas , A. M. . (2020). Estudos atuais sobre medicamentos para combater a COVID-19. Brazilian Journal of Implantology and Health Sciences , 2(11), 01-09. https://doi.org/10.36557/2674-8169.2020v2n11p01-09.

15. Coelho Paraguassu, E. . (2020). COVID-19, a relação direta entre o capital, solidariedade e as vidas. Brazilian Journal of Implantology and Health Sciences , 2(3), 01-04. Recuperado de https://bjihs.emnuvens.com.br/bjihs/article/view/33 